\title{
Filigrane
}

Écoutes psychanalytiques

\section{Le Centre de psychanalyse et de psychothérapie É. et J. Kestemberg. Une expérience thérapeutique entre psychanalyse et psychiatrie}

\section{Alain Gibeault}

Volume 27, numéro 2, 2018

La consultation psychanalytique aujourd'hui, entre héritages et remaniements

URI : https://id.erudit.org/iderudit/1055747ar

DOI : https://doi.org/10.7202/1055747ar

Aller au sommaire du numéro

Éditeur(s)

Revue Santé mentale au Québec

ISSN

1192-1412 (imprimé)

1911-4656 (numérique)

Découvrir la revue

Citer cet article

Gibeault, A. (2018). Le Centre de psychanalyse et de psychothérapie É. et

$\mathrm{J}$. Kestemberg. Une expérience thérapeutique entre psychanalyse

et psychiatrie. Filigrane, 27(2), 29-37. https://doi.org/10.7202/1055747ar
Résumé de l'article

Le Centre de psychanalyse et de psychothérapie É. et J. Kestemberg propose des conditions exceptionnelles de traitements psychanalytiques pour des patients présentant un fonctionnement psychotique, qui peuvent s'appuyer sur des tiers objectivés pour faire face à leurs menaces d'intrusion. La consultation psychanalytique au Centre avec un patient schizophrène suivi ultérieurement en psychodrame psychanalytique individuel permet d'illustrer la complémentarité entre les approches psychanalytique et psychiatrique dans le traitement de ces patients difficiles. 


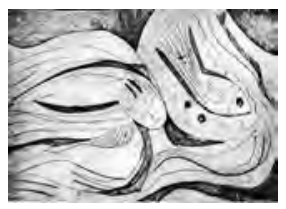

\title{
Le Centre de psychanalyse et de psychothérapie É. et J. Kestemberg. Une expérience thérapeutique entre psychanalyse et psychiatrie ${ }^{1}$
}

\author{
Alain Gibeault
}

\begin{abstract}
Résumé: Le Centre de psychanalyse et de psychothérapie É. et J. Kestemberg propose des conditions exceptionnelles de traitements psychanalytiques pour des patients présentant un fonctionnement psychotique, qui peuvent s'appuyer sur des tiers objectivés pour faire face à leurs menaces d'intrusion. La consultation psychanalytique au Centre avec un patient schizophrène suivi ultérieurement en psychodrame psychanalytique individuel permet d'illustrer la complémentarité entre les approches psychanalytique et psychiatrique dans le traitement de ces patients difficiles.
\end{abstract}

Mots clés: centre psychanalytique; personnage tiers; fonctionnement psychotique; consultation psychanalytique; tiers institutionnel.

\begin{abstract}
The Center for Psychoanalysis and Psychotherapy É. and J. Kestemberg proposes exceptional psychoanalytic treatment conditions for patients with psychotic functioning, who can rely on objectified third parties to cope with their threats of intrusion. The psychoanalytic consultation at the Center with a schizophrenic patient subsequently followed in individual psychoanalytic psychodrama illustrates the complementarity between psychoanalytic and psychiatric approaches in the treatment of these difficult patients.
\end{abstract}

Keywords: psychoanalytic center; third character; psychotic functioning; psychoanalytic consultation; institutional third party.

e Centre de psychanalyse et de psychothérapie Évelyne et Jean Kestemberg
a été fondé en 1974 par Jean Kestemberg, psychiatre et psychanalyste for-
mateur de la Société psychanalytique de Paris, et dirigé dès 1975 par son
épouse, Évelyne Kestemberg, psychologue clinicienne et également psycha-
nalyste formatrice de la Société psychanalytique de Paris, pour recevoir des
patients qui présentent des troubles psychotiques et des troubles graves de la 
personnalité. Le Centre fait partie des différentes institutions psychiatriques appartenant à l'Association de santé mentale du $13^{\mathrm{e}}$ arrondissement de Paris. Celle-ci a été créée en 1958 par un groupe de psychiatres et de psychanalystes soucieux d'apporter à une population démunie des soins gratuits en santé mentale dans une perspective conjuguant les approches psychiatrique et psychanalytique. Certains de ses membres fondateurs ont été des grands noms de la psychanalyse française, tous membres de la Société psychanalytique de Paris, en particulier Serge Lebovici et René Diatkine, qui ont fondé au sein de l'Association de santé mentale du $13^{\mathrm{e}}$ arrondissement le Centre Alfred Binet pour le traitement psychanalytique des enfants et des adolescents.

Le personnel est constitué d'un directeur psychanalyste, d'une cinquantaine de psychothérapeutes psychanalystes qui travaillent tous à temps partiel et d'une secrétaire à temps plein. Les psychothérapeutes sont pour un cinquième médecins psychiatres et les autres psychologues cliniciens. La file active est de 220 patients adultes: les trois quarts sont suivis en psychothérapie psychanalytique individuelle à raison d'une séance de 45 minutes par semaine, les autres sont suivis en psychanalyse, en psychodrame psychanalytique individuel et en psychothérapie psychanalytique corporelle.

La psychopathologie des patients a déterminé, pour une part importante, les modalités de fonctionnement du Centre qui, dès sa fondation, ont introduit un rapport nouveau entre psychiatrie et psychanalyse dans un souci de ne pas inféoder la psychanalyse à la psychiatrie ni de limiter la psychiatrie dans son évolution. Un choix était possible entre deux options: ou inclure les psychothérapeutes dans des équipes psychiatriques, ou créer un centre de psychanalyse et de psychothérapie dans un lieu distinct, qui offrirait au patient la possibilité de bien différencier la fonction psychiatrique de la fonction psychothérapique et ainsi de mieux se protéger de la menace anéantissante d'être englobé par un objet unique. Cette fonction tierce de l'institution n'aurait toutefois pas suffi sans le rôle du personnage tiers représenté par la fonction du Directeur. Celui-ci ne prend généralement pas lui-même de patients en traitement au Centre, mais il assure les premiers entretiens en présence de quelques collaborateurs qui n'interviennent pas entre le patient et lui. Ce cadre a pour spécificité de permettre au patient, souvent angoissé par la relation duelle, de s'appuyer sur la présence de ces collaborateurs afin d'alléger l'intensité affective de la rencontre.

Cette dimension du tiers analytique a été particulièrement développée par Évelyne Kestemberg qui évoquait la fonction d'un "personnage tiers» dans le parcours des patients adressés très souvent par les psychiatres ou les 
soignants de l'institution psychiatrique: «Quelle que soit la bonne relation avec cette personne ou cette institution tierce, l'action qui en émane n'est, ni au niveau du patient ni au niveau d'une possible élucidation personnelle de l'analyste, directement atteinte. Il s'est donc avéré nécessaire qu'à ce tiers non maniable en termes de compréhension théorique soit substitué, autant que faire se peut, un personnage tiers (entre l'analyste et le patient), dont le mode de fonctionnement puisse faire l'objet d'une connaissance élaborative et d'un maniement technique contrôlable» (1985, p. 148-149). Le personnage tiers évoque une médiation entre la personne et la fonction dans l'idée d'une représentation et d'un rôle à jouer. Il s'inscrit dans une fonction de médiation symbolisante et renvoie à une «nécessité processuelle» de l'agir (Donnet, 2005) dans la situation analytique, dans la transformation de la mise en acte en mise en scène, ou mieux, en mise en jeu.

Dans la même perspective, André Green a introduit ultérieurement «le concept de tiercéité» comme «une théorie de la triangulation généralisée à tiers substituable», sans qu'il faille pour autant ramener cette triangulation à la structure œdipienne: «Il est particulièrement possible d'envisager des relations triangulaires où le tiers ne représenterait pas la fonction paternelle. En revanche, il me semble y avoir intérêt à ne pas se laisser emprisonner dans la relation duelle» (Green, 2002, p. 267). De ce point de vue, la référence au tiers concerne la relation à l'objet absent. Cela signifie que toute structure ou événement psychique est caractérisé par les deux polarités du sujet et de l'objet, ce qui renvoie à «l'autre de l'objet», place qui dans la relation à la mère avec l'enfant peut être occupée par une personne différente du père, par exemple un membre de la fratrie de l'enfant ou de la mère, un objet du désir de la mère différent du père, l'un des parents de la mère, etc. Le concept de tiercéité donne de la valeur à la relation triangulaire en général, ce qui est très important dans l'approche du premier entretien et dans les différences possibles entre l'entretien préliminaire où le consultant envisage de prendre le patient en traitement ou de l'adresser à un autre collègue en restant une référence possible, comme dans la situation institutionnelle.

C'est à partir de cette conception du tiers que se sont organisées les modalités du premier entretien au Centre, mené par le Directeur en présence d'autres psychanalystes. Les patients qui ne supportent pas ce dispositif peuvent rencontrer le Directeur individuellement, ce qui est généralement le cas pour les patients qui le reverront ultérieurement au cours ou après la fin de leur traitement. L'expérience a montré que plus l'organisation psychique des patients est d'ordre psychotique, plus la présence des tiers 
ne les gêne pas et peut, au contraire, leur être utile. Inversement, plus les patients sont proches de la névrose, plus le dialogue singulier leur est nécessaire. Du fait de ce cadre, le Directeur joue donc un rôle essentiel de tiers pour le présent et l'avenir. Il est en effet important pour des patients aux organisations psychotiques complexes de pouvoir s'appuyer sur la possibilité de revoir le Directeur à des moments critiques de leur vie ou de la poursuite de leur cure, ce qui leur permet d'élaborer des angoisses inhérentes au travail psychothérapique individuel.

Ajoutons par ailleurs que le délai d'attente d'un premier entretien peut être de plusieurs mois, mais, à partir du moment où il a eu lieu, nous faisons en sorte que le traitement psychanalytique puisse commencer rapidement, au Centre ou en ville s'il s'agit d'une indication pour un travail psychanalytique en pratique libérale. Il faut préciser ici que ce délai d'attente n'est pas forcément négatif puisque, entre le moment où le patient a pris rendez-vous et le moment de la consultation, se fait souvent un travail important d'élaboration chez le patient dans la perspective d'une rencontre avec un psychanalyste. Évelyne Kestemberg insistait d'ailleurs sur l'importance de ce qui se passe avant le premier entretien, de ce qui surviendra ensuite pendant la consultation et enfin sur l'après du premier entretien eu égard à la possibilité d'entreprendre un traitement psychanalytique.

Le patient est souvent aidé dans ce travail psychique par le psychiatre qui a adressé le patient au Centre et qui poursuivra avec lui un travail d'accompagnement et de soutien. Il faut souligner ici que les patients psychotiques ou limites adressés au Centre ne sont jamais reçus dans un état de crise aiguë. C'est la fonction du suivi psychiatrique d'assurer si nécessaire médication et hospitalisation, et de juger de l'opportunité pour un tel patient d'envisager une consultation psychanalytique au Centre.

Avec l'autorisation des patients, les premiers entretiens font tous l'objet d'un enregistrement audio; les comptes rendus du consultant sont ensuite transcrits par la secrétaire et mis dans les dossiers des patients. Deux fois par mois, un premier entretien, effectué à des fins d'enseignement et de recherche, est transmis en direct par vidéo à un auditoire de professionnels de la santé mentale, toujours avec l'accord écrit du patient.

Ces premiers entretiens ont pour but d'évaluer l'économie psychique du patient à partir de six paramètres décrits par É. Kestemberg (1985) permettant de faire le choix entre différentes indications thérapeutiques: le degré de liberté intérieure du patient ou du moins son désir d'entrer en relation avec un analyste et, par voie de conséquence, avec lui-même; les possibilités 
de mobilisation psychique du patient dans cette situation d'entretien et d'écoute particulière; la qualité de l'histoire personnelle rapportée; les possibilités fantasmatiques et oniriques maintenues ou non; un aperçu de la répartition des investissements narcissiques et objectaux; l'observation d'éléments contre-transférentiels qui peuvent infléchir l'entretien dans un sens négatif ou positif.

La présence ou l'absence de ces éléments permet à l'analyste d'évaluer le processus du premier entretien et la qualité de l'économie psychique du patient à ce moment précis, de faire un possible pronostic et un choix parmi des propositions thérapeutiques différentes. Dans tous les cas, il s'agit que le premier entretien avec un psychanalyste soit l'occasion d'une rencontre. C'est d'ailleurs ainsi qu'un patient schizophrène que j'ai reçu en premier entretien a défini notre échange.

Ce patient schizophrène âgé de 28 ans est hospitalisé depuis quelques années lorsque je le rencontre pour la première fois. Lors du premier entretien, Jean est en hospitalisation d'office à l'hôpital psychiatrique depuis un an et demi. Il m’a été envoyé par son psychiatre le $\mathrm{D}^{\mathrm{r}} \mathrm{K}$. qui le reçoit depuis son arrivée au rythme d'une séance par semaine à l'hôpital. Au cours de ce suivi psychiatrique, il a été confronté à un discours extrêmement répétitif de ce patient et a pensé qu'un travail psychanalytique pourrait favoriser un changement psychique.

L'histoire de Jean est tragique. Âgé de 28 ans, Jean a vécu toute son enfance dans un petit village de province avec un père alcoolique et une mère qui se faisait sadiser par son mari. Il fait une première décompensation psychotique à 18 ans pour laquelle il a été d'abord hospitalisé en province et mis sous neuroleptiques. Après une amélioration de son état, il décide de venir à Paris pour y faire une formation professionnelle dans la restauration. Ne prenant pas régulièrement ses neuroleptiques, il fait une rechute avec épisode délirant qui entraîne une hospitalisation dans notre hôpital psychiatrique. Désorganisé dans sa pensée, il refuse le suivi psychiatrique proposé par le $\mathrm{D}^{\mathrm{r}} \mathrm{K}$. qu'il voit pour la première fois et à qui il pourra dire d'une manière énigmatique: «J'ai su vous trouver; je saurai vous retrouver».

Par la suite, il rend visite régulièrement à une vieille dame seule, Arlette, amie de sa grand-mère et du même âge, qui le nourrit et lui donne un peu d'argent pour acheter des cigarettes. La famille d'Arlette s'inquiète et l'un des gendres lui fait savoir leur désaccord. Dans ce contexte où il craint de perdre Arlette, il l'assassine à l'arme blanche. Interné pendant un an dans 
une Unité pour malades difficiles, il est ensuite transféré à notre hôpital, où il « retrouve» le $\mathrm{D}^{\mathrm{r}} \mathrm{K}$.

Au cours de l'entretien avec moi, Jean parle volontiers de sa solitude et de sa détresse, à l'origine de sa désorganisation psychique: ses pleurs pendant trois ans seul à Paris, l'angoisse catastrophique à l'idée de perdre la présence d'Arlette et la pulsion meurtrière à la tuer qu'il décrit comme «quelque chose qui s'engrène » et «un effet boule de neige» relatif à la mort successive et récente de ses proches, en particulier son père et ses grandsparents maternels. Grâce aux neuroleptiques, hallucinations et délires ont diminué, mais il conserve à l'hôpital une impression de déjà $v u$, un vécu hallucinatoire dont la fonction est de lutter contre une angoisse d'anéantissement qu'il décrit comme un blanc à certains moments de sa vie. Comme le remarque avec pertinence Racamier, «l'adjectif blanc évoque, par le jeu des images, à la fois la surchauffe économique et la vacuité fantasmatique [...]. Un évènement de cette nature et de cette grandeur fait vivre l'extase et produit une catastrophe: telle est la catastrophe psychotique» (1992, p. 171).

Alors que je souhaite évaluer sa capacité à rêver, il me rapporte un rêve récurrent de son enfance: «Des rêves, alors j'en ai eu depuis tout petit, tout petit, que je n'ai jamais oubliés de ma vie, c'était la chambre de mes parents, comme si, ici il y avait un lit et tout ça, il y avait des luminaires, vous voyez ce que je veux dire, des chandeliers, des luminaires au plafond, au mur, et je voyais du ketchup couler comme du sang, couler des murs, et c'est un rêve que j'ai fait tout petit, tout petit, je devais avoir 5 ans peut-être, 5 ans ou 6 ans et c'est quelque chose que je n'ai jamais, jamais, jamais oublié, c'est un rêve que je n'ai jamais oublié». Qu'en est-il du statut psychique de ce rêve récurrent de son enfance? Je suis sensible au fait que la scène primitive le renvoie immédiatement à une violence meurtrière: les parents sont morts, il en reste une trace visible sur le mur; du sang coule sur le mur. Ils se sont entretués ou plutôt ils ont été assassinés par le patient lui-même.

Ce patient a fait une décompensation schizophrénique à l'adolescence, et l'on peut penser que ce rêve, qui peut rappeler la scène d'un film d'horreur, est un rêve pré-psychotique proche des terreurs nocturnes de l'enfance, préfigurant, comme le suggère Racamier (1976), l'irruption psychotique, tout en en préservant le sujet. Le rêve est à la fois très dense et répétitif, et témoigne de la tentative du patient de maîtriser une angoisse d'anéantissement qui risque de le submerger. D'où l'hypothèse d'une capacité certes de figurer les enjeux de cette angoisse, mais d'une faillite de l'espace du rêve et de la fonction onirique. 
Jean évoque ensuite des cauchemars actuels dont il ne se souvient pas et dont il se demande s'ils peuvent avoir un lien par exemple avec l'entretien que nous allions avoir. Manifestement, l'entretien pouvait susciter une angoisse persécutoire qu'il a toutefois surmontée pour permettre un échange sur les enjeux de sa vie psychique.

Alors que je lui demande à la fin de notre entretien ce qu'il a pu en penser, il me répond avec pertinence: "C'est uniquement une rencontre... pas une connaissance, oui une rencontre». Nous nous sommes en effet rencontrés dans la possibilité qu'il a eue de pouvoir être reconnu dans ses affects, même les plus angoissants, et pour moi de comprendre les enjeux de son fonctionnement psychique. Il est vrai qu'il ne me connaissait pas et c'est justement ce qui pouvait susciter la crainte d'être confronté à une dimension d'inconnu, source de violence de la part d'autrui comme de sa part à luimême. Sensible à sa capacité à vouloir transmettre le tragique de son existence et le désir de vouloir comprendre sa réalité psychique, j'ai pensé qu'il pouvait bénéficier d'un travail analytique et je lui ai proposé alors de faire un psychodrame psychanalytique, dans lequel il s'est engagé six mois plus tard. Ce traitement qui se poursuit toujours au Centre depuis plus de dix ans a montré l'intérêt d'une consultation et d'un traitement psychanalytique qui s'appuie sur la complémentarité d'un suivi psychiatrique et hospitalier.

La possibilité que se développe un premier entretien puis un travail analytique suppose ainsi la référence au tiers. Il peut être virtuel dans le lien entre le cadre et le processus; le cadre analytique suppose de la part de l'analyste une attitude de neutralité bienveillante qui permet de passer de la séduction de l'analyste comme personne à la séduction de la méthode (Donnet, 1995), c'est-à-dire de l'investissement de l'analyste comme personne réelle à l'investissement de l'analyste dans sa fonction analytique. C'est même ce qui peut conduire à ne pas multiplier les premiers entretiens et de préférer les limiter à un ou deux seulement: la réalité perceptuelle de l'analyste ne doit pas empêcher la projection des imagos parentales.

La virtualité de ce tiers est généralement suffisante dans les organisations névrotiques. Dans les organisations non névrotiques (troubles graves de la personnalité et psychoses), il est souvent nécessaire que ce tiers soit objectivé dans le cadre d'une institution, par un premier entretien avec un consultant qui généralement ne prendra pas le patient lui-même en traitement ou encore avec la présence d'une assistance. C'est le propre des consultations dans un centre psychanalytique, comme le Centre de psychanalyse et de psychothérapie Évelyne et Jean Kestemberg, qui depuis sa fondation offre 
à la population du $13^{\mathrm{e}}$ arrondissement de Paris la possibilité d'entreprendre gratuitement un traitement psychanalytique sans limite quant à la durée de celui-ci. La proposition faite au patient de faire un don à l'institution permet éventuellement de négocier la dépendance parfois difficile que représente la gratuité, car c'est alors l'institution qui dépend de la contribution du patient. L'argent dans la cure, donné et/ou fantasmé, participe ainsi de la fonction symbolisante du tiers institutionnel.

La consultation dans une institution montre aussi qu'elle peut favoriser plus facilement l'élaboration des «mises en acte» des patients présentant un fonctionnement psychotique; la référence au tiers matérialisé permet en effet de mieux faire face «à l'orage émotionnel» suscité par la rencontre analytique et l'accès à « un changement de niveau» du conscient à l'inconscient, comme le montrent les travaux du Groupe de travail de la Fédération européenne de psychanalyse sur les enjeux du premier entretien (Reith et al., 2012).

Le premier entretien dans une institution montre l'intérêt du cadre fondé sur la différence entre un analyste consultant et un analyste traitant qui, comme le suggère Jean-Luc Donnet, permet de «rendre le transfert mieux transférable» (2013, p. 47). Cette situation est plus favorable pour analyser éventuellement une possible fixation et idéalisation, qui contribueraient à renforcer le déni et le clivage du Moi, comme cela peut être le cas dans la pratique privée. On peut penser que le dispositif du psychodrame psychanalytique s'appuie aussi sur la différence entre deux fonctions analytiques, celle du meneur de jeu qui ne joue pas et celle des psychodramatistes qui jouent des scènes dont le thème est proposé par le patient.

Ce dispositif de la consultation dans une institution ne serait donc pas un obstacle supplémentaire au développement d'un processus analytique, mais donnerait la possibilité de considérer les enjeux de la discontinuité dans l'expérience transférentielle. Si le processus analytique doit contribuer au développement de la symbolisation, le choix d'un cadre pour le premier entretien est relatif à l'évaluation du fonctionnement mental d'un patient et à la possibilité ainsi offerte de permettre l'expérience d'un «transfert sur la méthode et sur la parole», leur permettant de se «préparer au deuil futur sur l'objet» (Donnet, 2013). Le premier entretien est dès lors toujours lié au début comme à la fin du traitement analytique. La fonction d'un Centre de psychanalyse vise ainsi autant à offrir un traitement psychanalytique pour les plus démunis financièrement que pour ceux qui psychiquement pourraient rencontrer des difficultés supplémentaires pour engager un travail 
analytique leur permettant de surmonter leur détresse psychique autrement que par le recours au délire ou à des agirs auto- ou hétéro-destructeurs.

\author{
Alain Gibeault \\ alain.gibeault@gmail.com
}

\title{
Notes
}

1. Conférence présentée dans le cadre du Congrès de l'Association psychanalytique internationale (API/IPA), à Boston, les 22-25 juillet 2015, dans le panel «Le processus de consultation dans trois Centres de psychanalyse aujourd'hui (CCTP JeanFavreau, Centre Kestemberg, Clinique psychanalytique de Montréal): or ou cuivre? Sociothérapie ou psychanalyse?» (Geneviève Welsh, présidente; conférenciers: Alain Gibeault, Danielle Kaswin-Bonnefous et Isabelle Lasvergnas).

\section{Références}

Donnet, J.-L. (2013). La rencontre consultative. Dans J. Bouhsira et M. Janin-Oudinot (dir.), La consultation psychanalytique (p. 29-49). Paris: Presses universitaires de France.

Donnet, J.-L. (2005) La situation analysante. Paris: Presses universitaires de France.

Donnet, J.-L. (1995). Le divan bien tempéré. Paris: Presse universitaires de France.

Green, A. (2002). Idées directrices pour une psychanalyse contemporaine: méconnaissance et reconnaissance de l'inconscient. Paris: Presses universitaires de France.

Kestemberg, É. (1985). Quoi de neuf? Ou les enseignements du «Premier Entretien». Dans A. Amyot, J. Leblanc et W. Reid (dir.), Psychiatrie-psychanalyse. Jalons pour une fécondation réciproque ( $\mathrm{n}^{\circ}$ 10, p. 15-24). Chicoutimi: Gaétan Morin.

Kestemberg, É. (1981). Le personnage tiers. Sa nature - sa fonction. Dans auteur, La psychose froide (p. 145-177). Paris: Presses universitaires de France.

Racamier, P.-C. (1992). Le génie des origines. Psychanalyse et psychoses. Paris: Payot.

Racamier, P. C. (1976). Rêve et psychose: rêve ou psychose. Revue française de psychanalyse, 40 (1), 173-193.

Reith, B. et al. (2012). Initiating Psychoanalysis. Perspectives. New York: Routledge. 